\title{
SIMPLE RIEMANN FUNCTIONS
}

\author{
BY DAVID H. WOOD
}

Communicated by James H. Bramble, April 28, 1976

Research of Cohn [1] and Daggit [2] guesses or deduces functions $M$ and $G$ such that some Riemann functions have the form $R=M f(G)$. The Riemann function is then found by solving an ordinary differential equation for $f$. Roughly speaking, all the results of Riemann, Cohn and Daggit are simple Riemann functions because (i) the equation is almost selfadjoint, (ii) the ordinary differential equation for $f$ has coefficients depending on $G$, and (iii) the characteristic conditions for the Riemann function become initial conditions for $f$. These restrictions, formulated in Definition 2, seem mild because omitting any one of the three would allow every Riemann function to satisfy Definition 2 .

Surprisingly, these conditions lead directly to expressions for $M$ and $G$ (Theorems 1 and 2). With these, all of Daggit's results are derived (Theorems 3 to 7) from one of his hypotheses. For a selfadjoint equation to have a simple Riemann function, Daggit's hypothesis is sufficient as well as necessary (Theorem 8). This announcement therefore contains proof that all simple Riemann functions are known for selfadjoint equations. The proofs for the general case are given in another paper [4].

Definition 1. The unique Riemann function $R$ for the equation

$$
U_{x y}+A(x, y) U_{x}+B(x, y) U_{y}+C(x, y) U=0
$$

satisfies the equation adjoint to (1.1), depends on two parameters $X$ and $Y$, reduces to 1 at the point $(x, y)=(X, Y)$, and obeys

$$
R_{x}=B R \text { when } y=Y \text { and } R_{y}=A R \text { when } x=X .
$$

When a multiplier $M$ is chosen, substituting $R=M(x, y, X, Y) \times U(x, y, X, Y)$ into the equation adjoint to (1.1) gives

$$
U_{x y}+a(x, y, X, Y) U_{x}+b(x, y, X, Y) U_{y}+c(x, y, X, Y) U=0 .
$$

If a guess $G$ is then chosen, substituting $U=f(G(x, y, X, Y), X, Y)$ into (1.3) yields

AMS (MOS) subject classifications (1970). Primary 35L10, 35 L15; Secondary 35C05, $35 \mathrm{C} 99$.

Key words and phrases. Riemann function, Riemann-Green function, fundamental solution, Green's function. 


$$
\alpha(x, y, X, Y) f^{\prime \prime}+\beta(x, y, X, Y) f^{\prime}+f=0 .
$$

Definition 2. The Riemann function for (1.1) is a simple Riemann function if it has the form $R=M(x, y, X, Y) f(G(x, y, X, Y), X, Y)$ and for this $M$ and $G$, (i) the coefficients in (1.3) satisfy $a_{x}=-a b$, (ii) the coefficient $\alpha$ of (1.4) satisfies $\partial(\alpha, G) / \partial(x, y)=0$, which implies $\partial(\beta, G) / \partial(x, y)=0$, and (iii) $G(x, Y, X, Y)=G(X, y, X, Y)$, which is finite (otherwise $G$ could be replaced by $1 / G)$.

\section{THEOREM 1. Any $M$ is a function of $X$ and $Y$ times}

$$
M=\exp \left(\int_{Y}^{y} A(x, t) d t+\int_{X}^{x} B(s, Y) d s\right),
$$

and determines $a=0, b=\int_{Y}^{y} A_{x}(x, t)-B_{y}(x, t) d t$, and $c=C-A B-B_{y}$.

Proof for $A=B=0 . M$ is a fixed function of $X$ and $Y$ when $x=X$ or $y=Y$, because both $R$ and $G$ have this property. $M$ also satisfies the equation $M_{x y}=0$ that results from imposing the condition $a_{x}=-a b$ on the coefficients $a=M_{y} / M, b=M_{x} / M$ and $c=C+M_{x y} / M$. The formula given for $M$ is the solution of this Goursat problem.

REMARK. Daggit introduces this multiplier [2, p. 93] .

Lemma 1. No generality is lost by assuming $G_{x y}+b G_{y}=0$.

Proof for $A=B=0$. Since $b$ is zero and $G_{x} G_{y} / G_{x y}=\alpha / \beta$ is a function of $G$ by condition (ii), the lemma is just a restatement, in complex characteristic coordinates, of the classical result "A function $F$ has the same level curves as some harmonic function if $\nabla^{2} F /(\nabla F)^{2}$ is a function of $F^{\prime \prime}[3$, p. 228] .

Remark. Cohn also proves Lemma 1 for $A=B=0$.

Theorem 2. Any $G$ must be functionally dependent on

$$
G=\int_{Y}^{y} \int_{X}^{x} c(s, t) \exp \left(\int_{X}^{s} b(\theta, t, Y) d \theta\right) d s d t .
$$

Proof for $A=B=0$. Since $G_{x y}$ is zero and $\alpha=\left(G_{x} G_{y}\right) / c$ is a function of $G$, say, $1 / g^{\prime \prime}(G)$, it follows that $g(G)$ satisfies $[g(G)]_{x y}=c$. But the only solution of this equation satisfying condition (iii) is $g(G)=\int_{Y}^{y} \int_{X}^{x} c(s, t) d s d t$ (plus an inconsequential term depending only $X$ and $Y$ ).

REMARK. Equation (1.6) with $b=0$ is the basic assumption of Cohn's research on selfadjoint equations.

Theorems 3 to 7 are based on one of the conditions necessary for Daggit's method [4, Theorem 2, p. 96, Lemma A, p. 98]: namely, that $c,(\ln c)_{x y}$, and $b_{y}$ are proportional. This hypothesis is invariant under all changes of variables that preserve the canonical form of (1.1).

THEOREM 3. If $c=0$ or $c-b_{y}=0$, then (1.1) has a simple Riemann function given by $R=M(x, y, X, Y)$ or $R=1 / M(X, Y, x, y)$, respectively. 
THEOREM 4. If $b_{y}=0, c \neq 0$, and $(\ln c)_{x y}=0$, then (1.1) has a simple Riemann function,

$$
R=M J_{0}(2 \sqrt{ } U), \text { where } U=\int_{Y}^{y} \int_{X}^{x} c(s, t) d s d t
$$

THEOREM 5. If $(\ln c)_{x y}=0$ and $b_{y} / c$ is a nonzero constant $-1 / k$, then (1.1) has a simple Riemann function, with $R / M=L_{k}(U)$, a Laguerre function of $U=-\ln [M(x, y, X, Y) M(X, Y, x, y)]$.

Theorem 6. Assume $c \neq 0$, and $b_{y}=0$. Denote

$$
\left[c(x, y) c\left(x_{0}, y_{0}\right)\right] /\left[c\left(x_{0}, y_{0}\right) c(x, y)\right]
$$

by $Q$. If there exists a constant $k_{2}$ such that $1=1 / 2 k_{2}(\ln c)_{x y} / c$, then (1.1) has a simple Riemann function,

$$
R=M_{2} F_{1}\left(p, q ; 1 ; 1-Q^{-1 / 2}\right),
$$

where $p$ and $q$ are the roots of $x^{2}-x-k_{2}=0$.

Theorem 7. Let $b_{y} \neq 0$. Assume $c \neq 0$ satisfies

$$
1=k_{1} \ln (Q) / \ln [M(x, y, X, Y) M(X, Y, x, y)]
$$

for some constant $k_{1}$. If $b_{y} / c$ is a constant $k_{1} / k_{2}$, then (1.1) has a simple Riemann function, $R=M_{2} F_{1}\left(p, q ; 1 ; 1-Q^{-1 / 2}\right)$, where $p$ and $q$ are the roots of $x^{2}+\left(k_{1}-1\right) x-k_{2}=0$.

THEOREM 8. When $b_{y}=0,(1.1)$ has a simple Riemann function if and only if $(\ln c)_{x y}$ and $c$ are proportional.

Proof For $A=B=0$. If (1.1) has a simple Riemann function, it is a function of $G=\int_{Y}^{y} \int_{X}^{x} c(s, t) d s d t$, by Theorem 2 , and $\partial\left(G_{x} G_{y} / c, G\right) / \partial(x, y)$ is zero by Definition 1. Using these conditions, Cohn has shown [1] that $(\ln c)_{x y}$ and $c$ are proportional. Conversely, Cohn actually exhibits [1] the required simple Riemann functions when $(\ln c)_{x y}$ and $c$ are proportional.

\section{REFERENCES}

1. Harvey Cohn, A functionally stable iteration process for hyperbolic equations, $\mathrm{J}$. Mathematical and Physical Sci. 7 (1973), 341-347.

2. Edward Daggit, The use of infinitesimal transformations in predicting the form of the Riemann (-Green) function, J. Math. Anal. Appl. 29 (1970), 91-108. MR 40 \# 3070.

3. C. E. Weatherburn, Differential geometry of three dimensions, Vol. 1, Cambridge Univ. Press, New York, 1961.

4. David H. Wood, Finding simple Riemann functions (in preparation).

NORTH ATLANTIC TREATY ORGANIZATION, SACLANT ASW RESEARCH CENTRE, VIALE SAN BARTOLOMEO 400, 19026 SAN BARTOLOMEO, (LA SPEZIA) ITALY

Current address: Code 312, New London Laboratory, Naval Underwater Systems Center, New London, Connecticut 06320 